\title{
Niños y niñas andinos en el Perú: crecer en un mundo de relaciones y responsabilidades
}

Enfants andins au Pérou : grandir dans un monde de relations et de responsabilités

Andean children in Peru: Growing up in a world of relationships and responsibilities

\section{Patricia Ames}

\section{OpenEdition}

Journals

\section{Edición electrónica}

URL: http://journals.openedition.org/bifea/4166

DOI: 10.4000/bifea.4166

ISSN: 2076-5827

Editor

Institut Français d'Études Andines

Edición impresa

Fecha de publicación: 1 diciembre 2013

Paginación: 389-409

ISSN: 0303-7495

Referencia electrónica

Patricia Ames, « Niños y niñas andinos en el Perú: crecer en un mundo de relaciones y

responsabilidades », Bulletin de l'Institut français d'études andines [En línea], 42 (3) | 2013, Publicado el

08 diciembre 2013, consultado el 21 diciembre 2020. URL : http://journals.openedition.org/bifea/4166 ; DOI : https://doi.org/10.4000/bifea.4166

\section{BY NO ND}

Les contenus du Bulletin de l'Institut français d'études andines sont mis à disposition selon les termes de la licence Creative Commons Attribution - Pas d'Utilisation Commerciale - Pas de Modification 4.0 International. 


\title{
Niños y niñas andinos en el Perú: crecer en un mundo de relaciones y responsabilidades
}

\author{
Patricia Ames*
}

\begin{abstract}
Resumen
Este artículo se enfoca en los niños andinos de habla quechua que viven en comunidades rurales en el Perú. Propone mostrar cómo los niños son integrados a su grupo social desde muy pequeños y en qué medida su participación en sus diferentes ambientes sociales va creciendo. Los cambios en la vida de los niños ocurren durante diversos ritos o se concretan, de forma más sutil, en transformaciones graduales en sus roles y responsabilidades en el hogar. Estos cambios se definen por el aprendizaje de habilidades prácticas. Desarrollan de este modo una identidad, un sentimiento de pertenencia y responsabilidad que los hace miembros reconocidos y valorados por su familia y comunidad. El artículo contempla enriquecer las discusiones sobre la construcción de las relaciones sociales y el sentimiento de pertenencia desde la infancia temprana y a lo largo de esta.
\end{abstract}

Palabras clave: niños andinos, aprendizaje, sentimiento de pertenencia, trabajo, Perú

\section{Enfants andins au Pérou : grandir dans un monde de relations et de responsabilités}

\section{Résumé}

Cet article porte sur les enfants andins de langue quechua qui vivent dans des communautés rurales, au Pérou. Il se propose de montrer comment les enfants sont intégrés dans leur groupe social depuis leur plus jeune âge et dans quelle mesure leur participation est croissante dans leurs différents " environnements sociaux ». Les changements dans la vie des enfants interviennent lors de divers rites ou se concretisent, de manière plus subtile, par des transformations graduelles dans leurs rôles et

Investigadora en el Instituto de Estudios Peruanos (IEP), Horacio Urteaga 694, Lima 11; profesora en la Pontificia Universidad Católica del Perú (PUCP), Av. Universitaria s/n, Lima 32. E-mails: pames@ iep.org.pe; pames@pucp.pe 
responsabilités au sein de la maisonnée. Ces changements passent par l'apprentissage de compétences pratiques. Ils développent ainsi une identité, un sentiment d'appartenance et de responsabilité qui les transforme en membres reconnus et valorisés au sein de leur famille et de leur communauté. L'article envisage d'enrichir les débats sur la construction des relations sociales et le sentiment d'appartenance dès la petite enfance, et tout au long de celle-ci.

Mots-clés : enfants andins, apprentissage, sentiment d'appartenance, travail, Pérou

\title{
Andean children in Peru: Growing up in a world of relationships and responsibilities
}

\begin{abstract}
This article focuses on Andean Quechua speaking children living in rural communities in Peru. It aims to show how children are integrated into their social groups since very young and how their participation increases in their various "social environments". Changes in children' lives take place through different rites or are embodied, more subtly, in gradual transformations of their roles and responsibilities within their household. These changes are defined by the learning of practical skills. Thus, they develop an identity, a sense of belonging and responsibility that makes them become members recognized and valued by their family and community. The article aims to contribute to debates on the construction of social relations and the sense of belonging since early childhood and throughout it
\end{abstract}

Keywords: Andean children, learning, sense of belonging, work, Peru

\section{INTRODUCCIÓN}

La reciente discusión crítica sobre el tema de la infancia desde las ciencias sociales (Gaitán, 2006) ha resaltado dos puntos de central importancia para el tema de este artículo: el primero señala que, con frecuencia, se habla de la infancia como una categoría homogénea y estática, cuando, en la realidad, es uno de los momentos de la vida más fuertemente marcado por el cambio y la transformación, física y social. En este sentido, el trabajo antropológico en diversos lugares del mundo ha revelado un conjunto diverso de momentos de cambio en las vidas de los niños, algunos de ellos identificados como «ritos de pasaje» (Van Gennep, 1960), y otros asociados con procesos de aprendizaje cotidiano no necesariamente ritualizados (Rogoff, 1996). Una segunda crítica de esta renovada visión de la infancia ha enfatizado el rol de los niños como agentes sociales que participan de sus vidas y de aquellas de quienes los rodean de manera activa y significativa (James \& Prout, 1997). Aquí también la Antropología puede contribuir a una mejor comprensión de la infancia trazando las formas en que las relaciones sociales de los niños se desarrollan a lo largo del tiempo desde sus primeros años de vida.

Es desde estas dos consideraciones que puede entenderse mejor el propósito de este artículo enfocado en la vida de los niños y niñas de comunidades rurales quechuas en el Perú. Se abordarán en particular las cuestiones relativas a la integración de los niños andinos a su sociedad, la adquisición de autonomía, la interiorización de saberes, destrezas sociales y culturales así como a los ritos de pasaje que les hacen pasar de una categoría de edad a otra. Por lo tanto, nos interesa visibilizar los 
procesos de cambio que los niños quechuas hablantes atraviesan, el aprendizaje que adquieren y la manera como desarrollan sus competencias, principalmente en el ámbito doméstico y comunitario. En este sentido, tendremos en cuenta también su participación en la escolarización formal como práctica casi universal que implica nuevos momentos de cambio y rituales propios. Este documento busca identificar y entender el significado de estos procesos para los niños, sus familias y sus comunidades, a partir de diversas experiencias de investigación a lo largo de varios años en los Andes centrales, principalmente en las regiones de Cuzco, Apurímac y Ayacucho.

El artículo explora asimismo diversas etapas de la vida infantil: los rituales de los primeros meses y años de vida, el paso a una etapa «intermedia» en la niñez y la consolidación de su participación en la vida social hacia los 12 años. Se identifican algunos procesos clave para responder a las preguntas anteriormente planteadas en el contexto de la vida infantil rural andina: la iniciación al trabajo como parte de la economía del hogar y de la formación de la persona, el aprendizaje basado en la observación y la participación y las relaciones sociales de los propios niños, que se van generando y fortaleciendo a lo largo de la infancia. Todo ello implica un conjunto de valores para los mismos padres y niños que van más allá de las necesidades de supervivencia y que se relacionan con el desarrollo de un sentido de responsabilidad, identidad y pertenencia, los cuales son discutidos en la sección final. En un primer momento presentaremos los aspectos teóricos y metodológicos de esta investigación y seguidamente se mostrarán los resultados dando lugar a la discusión1.

\section{APROXIMACIÓN CONCEPTUAL}

En la Antropología, los cambios a lo largo de la vida han sido a menudo estudiados dentro de un marco que postula etapas lineales y un modelo de ciclo según el cual las etapas de la vida son universales (todos pasan a través de ellas), estrictamente ordenadas (en la misma secuencia para todos) y coherentes (los cambios son consistentes a través de diferentes dominios de la vida) (Johnson-Hanks, 2002: 866). Sin embargo, este modelo ha sido criticado pues simplifica el curso de la vida y oscurece la realidad social. En vez de ello, diversos antropólogos abogan por un modelo de trayectoria de vida como proceso, en el cual las etapas están permanentemente creándose, reconociendo que los eventos de vida varían en gran medida en tiempo, ritmo, orden y sincronización (Johnson-Hanks, 2002: 867). Este enfoque no niega la existencia de cambios, ritos de pasaje y transiciones en la

1 Parte de este artículo se publicó en Learning, Culture and Social Interaction, 2 (3), "Learning to be responsible: Young children transitions outside school", pp. 143-154, (C) 2013 y se utiliza con permiso de Elsevier. La traducción al castellano se llevó a cabo con la ayuda de una subvención del Centro Internacional de Investigaciones para el Desarrollo, Canadá, bajo la Iniciativa Think Tank. Agradezco la oportunidad de haber continuado desarrollando estas ideas gracias a los cursos de antropología de la infancia y antropología de la educación que tuve a mi cargo en la Pontificia Universidad Católica del Perú (PUCP) entre agosto de 2012 y julio de 2013. Debo un agradecimiento especial a los estudiantes de ambos cursos por compartir el entusiasmo en estos temas. 
vida, sino que alerta al investigador para que observe los procesos que posibilitan tales transiciones en vez de tomarlas como eventos naturales; las etapas de vida reconocibles son construcciones socioculturales más que hechos biológicos. Esto se aplica sin lugar a dudas al caso de la infancia, cuya duración y contenidos son social y culturalmente construidos, lo que da lugar a una gran variedad de infancias en un territorio culturalmente diverso como el que nos ocupa.

Los cambios en las vidas infantiles que más han llamado la atención de los antropólogos son aquellos marcados por rituales específicos, como el clásico «rito de pasaje» definido por Van Gennep (1960). El rito de pasaje se refiere a un momento ritual clave en la vida de la persona donde se lleva a cabo un cambio de estatus importante y socialmente reconocido. Aunque se ha dedicado mucha atención antropológica a los ritos de pasaje asociados con la pubertad y el paso o la transformación de niño/a en adulto/a, se ha atendido mucho menos la infancia temprana e intermedia, a pesar de la existencia de estudios en estos períodos (Lancy \& Grove, 2011; Lestage 1999). De otro lado, se ha observado que los cambios producidos a lo largo de la infancia no siempre están marcados de modo ritual, sino que muchas veces son procesos más sutiles, en los que se adquiere gradualmente los conocimientos y destrezas que permiten convertirse en un miembro adulto en el seno de su sociedad (Paradise, 2011; Spindler, 1993). Ello indica la importancia de interesarse en los procesos de aprendizaje de la vida cotidiana, en los cuales se dan las interacciones que permiten tales transiciones.

Estas consideraciones ayudan a comprender que la conceptualización utilizada en el presente estudio adopte un enfoque a partir del cual los «cambios en los individuos se asumen como inseparables de su participación en la actividad sociocultural» (Rogoff, 1996: 273). Los cambios individuales son estudiados no como hechos aislados sino como parte de procesos interpersonales y comunitarios. Dentro de este enfoque, se entiende el aprendizaje como una práctica socialmente situada, la cual involucra una participación progresiva del aprendiz en las prácticas culturales de su comunidad (Lave \& Wegner, 1991; Rogoff, 1990). Como las comunidades estudiadas son rurales, muchas de las prácticas socioculturales observadas incluyen la participación de los niños en el trabajo agrícola y doméstico como forma de aprendizaje. Por lo tanto, tomaremos también en cuenta la literatura sobre el trabajo de los niños, que incluye las actividades infantiles productivas y domésticas dentro y fuera del hogar, tanto pagadas como no pagadas. Señala igualmente aspectos tanto negativos como positivos, los cuales dependen en gran medida no solo del tipo de actividad sino también del contexto, de las relaciones y los valores que se asignan a las actividades infantiles (Cavagnoud, 2011; Woodhead, 2007; Bourdillon, 2006; Boyden et al., 1998).

\section{PARTICIPANTES Y METODOLOGÍA}

En este artículo se utiliza información colectada en diversos estudios realizados en comunidades de los Andes centrales, en las regiones de Cuzco, Apurímac y Ayacucho. En la región Cuzco, tuve oportunidad de estudiar diversos temas, por 
tres años consecutivos, en una comunidad de la provincia de Paucartambo a finales de los años 1990 (Ames, 2001; 2002). En la región Apurímac se realizaron estudios de caso en una comunidad en dos años consecutivos (2007 y 2008) como parte del proyecto Niños del Milenio22. En la región de Ayacucho también realizamos estudios de caso en el año 2012 como parte de un estudio sobre la violencia infantil. En estos estudios participaron 38 niños y niñas (en similares proporciones) de entre 5 a 12 años de edad, todos ellos hablantes maternos del quechua.

La metodología empleada en todos los estudios fue de carácter cualitativo, con un enfoque etnográfico que buscaba captar tanto el contexto más amplio de la vida infantil como sus interacciones y experiencias más específicas. Utilizamos la observación participante en el hogar, la escuela y la comunidad de los niños así como entrevistas a profundidad con sus padres y madres. Adicionalmente, los niños participaron en sesiones grupales participativas y entrevistas individuales. Los padres y madres de los niños de Apurímac y Ayacucho ayudaron a reconstruir las biografías de los niños pequeños y a dar detalles sobre los cambios experimentados en el pasado, mientras que en el Cuzco nos concentramos en sus tareas presentes. Al menos 62 adultos participaron en entrevistas colectivas y/o individuales en las tres regiones. Las entrevistas fueron grabadas con el consentimiento de los participantes y se transcribieron para el análisis posterior. Las sesiones grupales y las observaciones fueron reportadas a través de recuentos narrativos basados en notas de campo llevadas a cabo durante la actividad o poco después. Las grabaciones sonoras y las fotografías de la actividad apoyaron los recuentos narrativos. La autora dirigió los equipos de trabajo que recogieron la información; en dicha capacidad, llevé a cabo directamente algunas entrevistas y observaciones, mientras que otras fueron realizadas por mis colegas. Todos los miembros del equipo usaron los mismos instrumentos semiestructurados de recolección de información (guías de entrevista, protocolos de observación y actividades grupales) y formatos de reporte para garantizar uniformidad y comparabilidad. Para cada estudio de caso, las diferentes fuentes de información fueron reunidas y analizadas, creando una comprensión más rica de la biografía de cada niño y permitiendo al mismo tiempo comparaciones entre ellos. Tras un análisis temático, seleccioné a un niño y una niña representativos del grupo de Andahuaylas para ofrecer una narrativa más detallada e ilustrativa de algunos procesos a los que hago referencia en este artículo.

2 Niños del Milenio (www.ninosdelmilenio.org), conocido internacionalmente como Young Lives (www.younglives.org.uk), es un estudio internacional que analiza los cambios referentes a la pobreza infantil siguiendo la vida de 12000 niños y niñas en cuatro países (Etiopía, India, Perú y Vietnam) a lo largo de 15 años. El financiamiento de Niños del Milenio proviene del Departamento de Desarrollo Internacional (DFID) del Reino Unido para el beneficio de países en vías de desarrollo. El estudio es actualmente cofinanciado por el Ministerio de Relaciones Exteriores de Holanda. La Fundación Bernard Van Leer y la Fundación Oak financian algunos de los estudios del proyecto. Los puntos de vista expresados en el texto corresponden a la autora. No pertenecen ni son necesariamente compartidos por Niños del Milenio/Young Lives, la Universidad de Oxford, DFID u otros patrocinadores. 


\section{ESCENARIOS DE LA INVESTIGACIÓN}

La población quechua hablante constituye el grupo indígena más grande del Perú: el último Censo Nacional (2007-INEI, 2008) señala un total de 3261750 hablantes, que representa el $83 \%$ del total de la población indígena del país. La misma fuente indica que los niños quechua hablantes muestran tasas de matrícula escolar similares (93\%) al promedio nacional (95\%) para el grupo de edad de seis a once años, mostrando que la educación formal se ha convertido en una experiencia cotidiana para casi la totalidad de niños de habla quechua. Sin embargo, ello se aplica sobre todo desde los seis años en adelante. Los niños quechua hablantes tienen un menor acceso a la educación inicial: mientras que solo un tercio (34 \%) de los niños de 3 a 5 años se encontraban matriculados en el nivel inicial en el 2007, más de la mitad de los niños hispano hablantes de la misma edad (55\%) lo estaban (Unicef, 2010). Cuando llega el momento de asistir al primer grado de la educación primaria, a la edad de seis años, el porcentaje se incrementa a un $80 \%$ para los niños quechua hablantes, lo cual representa una tasa más baja que los niños hispano hablantes de la mismo edad (91,6 \%) (UNICEF, 2010). Todo ello nos indica que las primeras experiencias de escolarización de los niños pequeños cuya lengua materna es el quechua pueden ser muy variadas y diferenciadas, aunque finalmente todos transiten por la educación primaria.

Por otro lado, los hogares indígenas en el Perú viven en condiciones de pobreza y pobreza extrema en mayor proporción que los hogares no indígenas: en 2009, $78 \%$ de los niños indígenas vivían en situación de pobreza, casi el doble del porcentaje de aquellos que tienen al castellano como su lengua materna, entre los cuales la pobreza llega al $40 \%$. La brecha en términos de extrema pobreza es aún mayor, ya que según la En cuesta Nacional de Hogares (INEI 2010a), el 12 \% de los niños no indígenas viven en dicha condición, mientras que esto ocurre con el $45 \%$ de los niños indígenas. Así, aunque el Perú está considerado actualmente como un país de ingreso medio (usando la clasificación del Banco Mundial, 2012), la riqueza está muy desigualmente distribuida. El 60 \% de la población rural vive en situación de pobreza en contraste con el 21 \% de la población urbana (INEI, 2010b). Las comunidades rurales estudiadas presentan por ello altos niveles de pobreza. En Ayacucho y Andahuaylas todas tenían acceso a servicios básicos como electricidad y agua entubada (excepto una, que usa agua de pozo). En Cuzco sin embargo, y a fines de los años 1990, la comunidad de Paucartambo no poseía electricidad. Todas tenían acceso a la educación inicial y primaria, si bien con modalidades diferentes (unidocente, multigrado, polidocente completa). En dos comunidades de Ayacucho existe un centro de estimulación temprana de reciente creación. Solo dos comunidades, las de mayor tamaño (una en Andahuaylas y una en Ayacucho), cuentan con educación secundaria y servicio de salud básica (posta

3 Las estadísticas oficiales en el Perú definen la condición de indígena a partir de la primera lengua que la persona aprendió en su niñez (lengua materna). 
médica). Las comunidades se describen brevemente a continuación en orden alfabético ${ }^{4}$.

Andahuaylas es una comunidad campesina ubicada entre 3000 y 3500 msnm, a unos 30 minutos de la capital distrital. Cerca del 96 \% de su población tiene al quechua como su primer idioma. Las familias siembran mayormente tubérculos y cereales andinos, y venden parte de sus cosechas. La mayoría de las madres tiene un bajo nivel educativo — $96 \%$ de ellas no ha finalizado la escuela primaria, y algunas no han asistido nunca a la escuela-. Más del 90 \% de la población vive en situación de pobreza (información de la segunda ronda de Niños del Milenio).

En Cangallo se visitaron cuatro comunidades campesinas ubicadas entre los 2900 y 4000 msnm, entre 10 y 45 minutos en transporte público de la capital distrital. Varían en tamaño, siendo la más pequeña de unas 60 familias, y la de mayor tamaño de 250 familias. En todas las comunidades la población es bilingüe quechua castellano. Ha sido una zona muy golpeada por el conflicto armado interno (1980-2000), con procesos de desplazamiento y retorno. La actividad económica predominante es la ganadería vacuna y la agricultura de diversos productos de acuerdo a las tierras de cada comunidad (papa, avena en las más altas; maíz, frejoles, calabaza en las que poseen laderas más bajas). Otras fuentes de ingreso monetario son la venta de madera de eucalipto, así como el empleo como mano de obra en campos agrícolas o minas cercanas.

Finalmente, Paucartambo es una comunidad campesina ubicada entre 3000 y 3700 msnm. Cuenta con 287 habitantes, cuya lengua materna es el quechua. Tiene poco contacto con la capital regional y provincial, si bien se ubica cerca de la carretera que conduce a ambas, a 8 y 1 hora de camino respectivamente, con escaso transporte público (un solo servicio al día). La población siembra mayormente cereales, tubérculos y algo de maíz en la zona baja y cuenta con rebaños de ovejas.

\section{INTEGRANDO A LOS NIÑOS DESDE LOS PRIMEROS AÑOS}

En esta parte se presentan los resultados ordenados en tres partes, correspondientes a grandes rasgos a las principales etapas identificadas en las vidas de los niños y niñas andinos quechua hablantes: la primera etapa desde el nacimiento al inicio de la autonomía que culmina hacia los 3 o 4 años, la etapa entre los 4 y 7 años donde se observa el paso a una fase intermedia de la niñez, que culmina hacia los 10 u 11 años y finalmente la etapa situada hacia los 12 y 13 años cuando los niños muestran una participación completa en la vida familiar y productiva.

4 Se utilizan seudónimos para los lugares y las personas, para proteger el anonimato de los participantes de la investigación. Los nombres de las comunidades se refieren a las provincias a las cuales pertenecen. 


\section{1. Rituales de crecimiento: iniciando la vida en sociedad}

La integración de los niños andinos a su sociedad se ha abordado desde la etapa previa al nacimiento (Del Pino et al., 2012; Platt, 2001; Lestage, 1999) y en relación a los ritos de pasaje. Durante los primeros 5 años de vida identificamos diversos momentos de celebración ritual que marcan el paso del tiempo y el cambio en la vida de los niños y niñas quechuas.

La información recolectada en el caso de Andahuaylas nos permite abordar estas cuestiones, pues uno de sus ejes tuvo el propósito de identificar las principales transiciones por las que atraviesan los niños, definidas como «eventos y/o procesos clave que ocurren en períodos o puntos de quiebre específicos durante el curso de la vida» (Vogler et al., 2008). Así, encontramos que la primera transición social de un niño o niña ocurre poco después del nacimiento y consiste en una ceremonia sencilla para darle un nombre: una semana después de nacer, el niño pasa por una especie de «pre-bautismo» (conocido también en la literatura como unuchaku, yacuchan o «agua de socorro»). Una persona es escogida como madrina o padrino para elegir un nombre y decirlo en voz alta, dejando caer agua sobre la cabeza del niño. Este «bautizo andino» no solo nombra al niño (otorgándole existencia social) sino que además busca proteger al recién nacido de riesgos sobrenaturales. También establece una primera relación social fuera de su familia nuclear al relacionarlo con un padrino o madrina. Lestage (1999) reporta el mismo rito en Laraos, Yauyos, enfatizando el carácter protector del mismo, al igual que Bolin (2006). Asimismo señala su existencia en otras comunidades como Tupe (Lima), Chía (Puno) y Santa Ana (Huancavelica).

Después del nacimiento, los bebés en Andahuaylas pasan sus primeros meses envueltos y fajados, en las espaldas de su madre, cargados en una manta o lliclla hasta que sean capaces de sentarse, pararse y caminar. Dar de lactar es común por los primeros 12 a 24 meses, o hasta que llega un nuevo bebé, y se cuida mucho a los niños por los primeros dos años, durante los que se mantienen muy cerca de su madre. Durante esta etapa, tanto niños como niñas son llamados indistintamente wawa, una palabra que no indica género ${ }^{5}$. La literatura sobre la infancia andina señala que los bebes más pequeños son considerados inmaduros, sexualmente indiferenciados y especialmente vulnerables en tanto su alma no está bien adherida a su cuerpo (Isbell, 1997; Suremain 2010). Quizás por ello durante su desarrollo se enfatiza la adquisición progresiva de la madurez y la humanidad y con ello la diferenciación sexual (Isbell, 1997).

Cerca de los dos o tres años ocurre otro rito importante, el primer corte de pelo6: en una ceremonia donde la familia y los amigos se reúnen, los invitados cortan un mechón del cabello del niño y dejan dinero o regalos a cambio. Se elige un padrino o madrina para esta ocasión y es el primero en cortar el cabello de su ahijado (y el

5 Aunque se puede acompañar de otra que lo precise, por ejemplo warmi wawa, bebe mujer.

6 Conocido en otras zonas como rutuy chicuy o chukcha rutukuy. 
más generoso con sus regalos). El primer corte de pelo indica el final de una fase: los niños pequeños ya no son bebes y dejan de estar con la madre la mayor parte del tiempo; comienzan a socializarse con otros, uniéndose a grupos de hermanos y parientes. El primer corte de pelo ha sido asociado en la literatura previa en otras partes de los Andes con el destete (Lestage, 1999; Román de Silgado, 1990) o con el empezar a caminar (Bolin, 2006; Isbell, 1997). Ortiz (2001) señala el inicio de una existencia social y de relaciones sociales propias, e Isbell (1997) indica que se recibe una primera herencia. Suremain (2010), en su estudio de poblaciones de habla quechua en la Amazonia boliviana, señala más ampliamente que este rito de paso engloba todo lo anterior, marcando el desarrollo de competencias sociales y motrices (hablar, caminar, alimentarse autónomamente) que indican el fin de una etapa, la infancia temprana, el inicio de una mayor autonomía, y la ruptura de un vínculo particularmente cercano con la madre. Varios autores (Suremain, 2010; Bolin, 2006; Ortiz, 2001; Lestage, 1999; Isbell, 1997) asocian el corte de pelo con el cambio de vestimenta: cuando los niños empiezan a caminar, tanto varones como mujeres, usan una especie de faldilla, denominada wali o wara, mientras dura el aprendizaje de control de los esfínteres (van desnudos bajo la faldilla). Cuando los bebés dejan de ser tales sin embargo, pasan a llevar ropas similares a las del varón y mujer adulto según su género. Una década atrás, era posible observar esta práctica en la comunidad cuzqueña de Paucartambo. En Andahuaylas, sin embargo, los niños y niñas ya no usan esta faldilla sino ropas de bebé, comercialmente producidas, pero cuando dejan de ser wawas usan vestimentas más parecidas a las formas en las que los varones y mujeres adultos se visten en su comunidad, marcando así su identidad sexual.

Finalmente, otra ceremonia que se lleva a cabo durante la infancia temprana en algunas familias de Andahuaylas es el bautismo católico. A través del bautismo, un niño es admitido a la Iglesia Católica —y a nuevas relaciones sociales—, pues se escogen nuevos padrinos y comienza una relación de obligaciones mutuas a lo largo de la vida entre el niño y sus padrinos. Como el bautismo católico se celebra con una fiesta costosa, no todos los niños son bautizados cuando bebés y la familia debe ahorrar un capital significativo antes de organizarlo. No todos los niños en Andahuaylas pasan por este rito porque varias familias son evangélicas.

En Andahuaylas nos fue posible observar y recoger la vigencia de ciertos rituales que marcan el cambio en la vida de los niños pequeños: su llegada e incorporación a una familia, el dejar de ser bebé y la dependencia cercana de la madre. La edad biológica se ha indicado apenas como referencia, pues se presta mayor atención a lo que los niños son capaces de hacer (caminar y desplazarse autónomamente, alimentarse solos, etc.). Otros estudios recientes en Ayacucho (Del Pino et al., 2012) han identificado umbrales de desarrollo más detallados en los primeros meses de vida, que dan cuenta de los cambios que van viviendo los bebés. Del mismo modo, nuestro estudio en Cangallo recogió información de las madres sobre los cambios en los primeros meses de vida (sentarse, levantar la cabeza, empezar a comer, etc.), que detalla la atención permanente hacia el desarrollo de los niños pequeños. 
Las prácticas que observamos marcan la progresión del niño o niña por la vida; son por ello parte de un «modelo autóctono (folk) de desarrollo infantil» como Lancy (2010) lo denomina. Es necesario reconocer esta sistematicidad en la crianza infantil para dejar de considerar estos modelos como un conjunto de costumbres exóticas y más bien reconocerlos como parte de un conocimiento local coherente sobre el desarrollo infantil. Así por ejemplo, la investigación ha demostrado que el uso de la manta y el fajado para cargar a los bebés en la espalda en los Andes protege a los niños pequeños de los factores de estrés de un ambiente de gran altitud (temperaturas frías, sequedad, baja presión de oxígeno) y conserva su energía, mientras que al mismo tiempo los ayuda a adaptarse progresivamente a las características del ambiente (Tronick et al., 1994). El uso de la faldilla facilita asimismo el proceso de control de esfínteres, si bien se ha reemplazado con el de los modernos pañales. Esto último muestra que estos modelos no son estáticos y que se transforman con los cambios en el entorno. Finalmente cabe resaltar que, a través de los ritos descritos, los niños inician, fortalecen o expanden sus relaciones sociales, al adquirir una relación de largo plazo con sus padrinos (de nombre, de bautizo, de corte de pelo), que involucra obligaciones mutuas y la oportunidad de acceder a recursos y apoyo, material y social. Sin embargo, se observa una importante heterogeneidad; no todas las familias siguen actualmente estas prácticas y algunas costumbres van desapareciendo, mostrando el impacto de nuevos modelos introducidos en las sociedades rurales.

\section{2. Aprendiendo a ser responsable: una inclusión progresiva en la vida social}

El desarrollo de la autonomía infantil adquiere mayor importancia aún en los años subsiguientes, y se liga a los procesos que les permiten interiorizar saberes y destrezas sociales y culturales. A partir de los 4 y 5 años ocurre un cambio gradual: la inclusión de los niños y niñas en un amplio rango de tareas, que se acompaña de evoluciones en sus roles y responsabilidades dentro de sus hogares. En Andahuaylas, se pudo observar este cambio progresivo que evidenció la forma como los niños pasaron de ser «niñitos» a los que se cuidan, a niños más «grandes» que son capaces de cuidar a otros y asumir una mayor diversidad de actividades. En efecto, los niños fueron asumiendo más responsabilidad en sus tareas domésticas y productivas así como desarrollaron habilidades que los preparaban para ser miembros productivos de sus hogares. Una mujer indicó que este proceso era posible porque hacia los 5 años los niños «se vuelven más juiciosos» y por ello son capaces de asumir responsabilidades. Este proceso posibilita una participación casi total en las actividades propias de la producción doméstica cerca de los $7 \mathrm{u}$ 8 años, aunque algunas tareas (usualmente aquellas que involucran mayor fuerza física y destreza) tenían que esperar hasta los 10 u 11 años. Estas expectativas fueron confirmadas por observaciones y entrevistas con los niños de mayor edad (12 y 13 años), mientras que la observación de los niños menores mostró el inicio de dicho proceso. En el caso del Cuzco, donde reconstruimos las actividades 
infantiles a distintas edades, pudimos comprobar un patrón similar, con los niños y niñas de 4 a 6 años colaborando con el hogar, y con los de 10 a 12 ya plenamente responsables de un conjunto de tareas domésticas y productivas.

En Cangallo encontramos puntos de vista similares: las mujeres señalaron que en los primeros cuatro años de vida los niños no podían ser castigados porque «no piensan», «no saben». Hacia los 5 años se considera que es posible exigirles una mayor participación en las responsabilidades domésticas. Como el estudio en Cangallo se enfocaba en la violencia infantil, se comprobó que junto al surgimiento de una mayor responsabilidad en los niños, se iniciaba también el uso del «chicote». Por ejemplo, una mujer indicó que a los cinco años ya se podía castigar a un niño porque a esa edad ya «se daba cuenta» de las cosas que hacía (y dejaba de hacer). Otra señaló que «ya se les explica porque ya entienden que cosas está bien o mal», aunque otra consideró que sus hijos no necesitaban ser castigados, «son para puro cariño no más» explicando que si les gritaba era peor porque hacían las cosas mal. Encontramos entonces discursos diferenciados respecto al uso del castigo físico, pero también identificamos su presencia y su relación con la asunción de nuevas responsabilidades.

La exigencia de colaborar deja de ser en cierto punto un juego, como puede haber sido en los años anteriores, y en caso de no cumplirse, se emplean castigos, físicos o verbales. Antes de los 4 o 5 años, como los niños «no entienden», es «por gusto» castigarles. Así, en todas las comunidades podíamos ver a niños de 2 o 3 años cargando un pequeño balde de agua, o unas ramitas para la leña, pero esto consiste en una ayuda voluntaria, un juego de imitación, más que en una responsabilidad asignada. Eso cambia progresivamente después de los 4 años, cuando empieza a convertirse en exigencia. Así, se reconoce que a los 4 años el niño «ya tiene responsabilidad de cuidar animalitos, ovejas (...) de dar comida a las gallinas». A partir de los 6 años, se reporta que los niños colaboran trayendo agua de la acequia, cargando leña, siguiendo a los animales, cuidando al bebé; ya son independientes y si no cumplen con las responsabilidades asignadas o desobedecen son castigados con ortiga, golpe o chicote.

Esta constatación nos llevó a revisar las informaciones de Andahuaylas, donde comprobamos que el castigo físico aparece como un correctivo cuando los niños incumplen las tareas y responsabilidades asignadas, al igual que en Cangallo, y que esto sucede más bien a partir de los 5 o 6 años. En la literatura encontramos que efectivamente entre los 5 y 10 años de edad los niños andinos están en una categoría de edad claramente diferente a la previa, en la que se enfatiza su identidad sexual y su participación en le mundo del trabajo (Isbell, 1997). Suremain (2010) asimismo indica que este proceso es progresivo y se inicia en los años posteriores al corte de pelo. Dos estudios de caso, un niño y una niña, provenientes de Andahuaylas, ilustran esta participación creciente de los niños en el trabajo doméstico y agrícola así como el tipo de aprendizaje que ocurre al realizarlo. 


\section{2. 1. Ana}

Conocí a Ana cuando tenía 5 años. Era la segunda de tres hijas y vivía con sus dos padres. Observé a Ana ayudando a su abuela con el ganado y acompañando a su madre a pastorear el rebaño, una actividad reservada a las mujeres en su comunidad. Ana reportó que además ayudaba con la cocina, el lavado de ropa y la alimentación de las gallinas y los cuyes. Durante nuestra visita al año siguiente, cuando Ana había empezado el primer grado, también comentó que, además de las actividades mencionadas, cuidaba a su hermana menor y ayudaba a cosechar papas. Su madre también reconoció los cambios y las nuevas actividades que Ana estaba asumiendo.

Madre de Ana: El año pasado todavía no se daba cuenta de nada, porque era pequeñita. (...) Trae agüita, cualquier cosita trae, cuando le decimos que traiga, va a comprar, está cambiando señora. (...)

Entrevistadora: ¿Y ahora hace algo por dinero?

Madre de Ana: Para dinero señora, sembramos verduras, luego lo llevan a la feria para ella misma. (...) Ana, con mi Ofelia. (...) aquí a la feria [de la comunidad]. (...) A eso se dedican, quieren vender, con los animales no mucho. (...) Para este viernes ya están preparando, para que vendan el sábado.

Entrevistadora: ¿Y ahora están jugando más o menos que el año pasado?

Madre de Ana: Ahora señora les gusta vender.

Aunque Ana había empezado a apoyar las actividades domésticas y productivas de su familia cuando la visitamos por primera vez, al año siguiente había consolidado y expandido su participación en un rango de actividades que eran parte de la vida diaria de su hogar, incluso ganando algo de dinero por su cuenta. Su madre indicó que era más «consciente» de las cosas. Esta actitud parece marcar la transición hacia nuevas responsabilidades. De hecho, ser consciente de las necesidades de los demás permite a los niños desarrollar la disposición a colaborar con otros. La consciencia también parece fundamental para el tipo de aprendizaje que tiene lugar durante esta transición. En efecto, cuando se preguntó sobre quién enseña a los niños y cómo lo hace, la respuesta más usual de los niños y los padres era que aprendían de sus padres y madres:

Entrevistadora: ¿Y en tu casa [qué haces]?

Ana: Cocinar. (...) Freír papa profesora.

Entrevistadora: ¿Y quién te enseñó a hacer eso?

Ana: Mi mamá profesora.

Entrevistadora: ¿Y te gusta hacer eso?

Ana: Sí profesora.

Este aprendizaje ocurre usualmente al observar y ayudar a los padres en actividades cotidianas, como explicó la madre de Ana al preguntarle sobre cómo aprendía Ana: 
Así cuando hacemos, [ella] nos mira cuando tejemos para que nos pueda seguir (...) Así hace también la mayorcita... En la cocina también ya sabrá, ellas nomás ya harán lo que preparamos, mirando, ellas prepararán.

El tipo de aprendizaje del que da cuenta la madre de Ana se aplica también a otros niños de su comunidad. Para que esto ocurra, se necesita una motivación intrínseca en la persona que aprende (para mirar a la gente, prestar atención), interés por aprender la tarea que se está desarrollando e identificación con quienes la realizan. García (2005) señala que en las poblaciones quechua hablantes el aprendizaje es considerado como una responsabilidad de quien aprende: pueden tener la ayuda de otros miembros de la comunidad, pero en última instancia el aprendizaje es una responsabilidad personal, pues la sociedad da la oportunidad de acceder a todas las situaciones de aprendizaje observando, imitando y ayudando. Por ello los intentos organizados de enseñar a los niños son poco usuales, como también ha sido observado en otros contextos no occidentales (Lancy, 2010). Las principales personas en este proceso son los mismos padres y hermanos mayores, y así como Ana estaba aprendiendo con su madre. En esta etapa, los niños varones empiezan a aprender con sus padres, como el caso de Felipe lo muestra a continuación.

\section{2. 2. Felipe}

Felipe tenía 5 años en nuestra primera visita. Vivía con sus padres y cuatro hermanos. En esta oportunidad, acompañaba a su madre o a su hermana mayor en sus visitas a los cerros cerca de la comunidad para pastorear el rebaño o trabajar en la chacra. Ayudaba también en el hogar con la cocina, la lavandería, la alimentación de los animales y recogía agua y leña. Durante el año antes de la segunda visita, Felipe expandió sus actividades ayudando a su padre en la chacra y usando una nueva herramienta en miniatura que su padre había confeccionado especialmente para él. Reportó tener nuevas actividades agrícolas que disfrutaba:

Entrevistadora: ¿Y tú, ya agarras trigo o no agarras trigo?

Felipe: Sí, con segadera.

Entrevistadora: ¿Y te gusta hacer eso?

Felipe: Sí... tengo pico [también].

Entrevistadora: ¿Quién te ha enseñado?

Felipe: Mi papá.

Felipe enfatizaba las habilidades agrícolas y el aprendizaje brindado por su padre. Aunque todavía pasaba gran parte de su tiempo con su madre, estaba experimentando el comienzo de la diferenciación de roles de género, especialmente en relación con el trabajo productivo. Eso se observa entre los niños mayores de su comunidad a través de una mayor dedicación de los varones a la chacra y de las niñas al pastoreo y a las actividades domésticas. En el caso de Felipe, dejó de ser un niño pequeño cuando su madre ya no lo tenía que cuidar:

El otro año en su jardín yo no más le cuidaba más. (...) Ahora desde que va a la escuela ya lo he dejado, mamá (...) Ya es mayorcito, mamá. (...) El 
carro le puede pisar a mi hijo diciendo decía [refiriéndose a la carretera] (...) Ya no, ahora ya le dejo [ir solo], ya sabe por dónde venir (...) Juega en el barrio, en las aguas juega en el jardín [inicial] mamá. (...) Ahora ha cambiado, desde que está en primer grado. Yo decía, «igual jugará decía», ahora ya no juega señorita, ya regresa limpio.

Las madres de Felipe y de Ana reconocen que sus hijos estaban desarrollando nuevas competencias y autonomía: se estaban volviendo más capaces de cuidar de sí mismos. También se encargaban de sus hermanos menores como observamos al acompañarlos a pastar: Felipe acompañaba a sus hermanas mayor y menor, de 11 y 4 años respectivamente, y a su tío de 3 años. De camino a los pastos, Felipe, entonces de 6 años, cuidaba a los niños pequeños, tomándolos de las manos en algunas partes difíciles del terreno o ayudándolos a levantarse si se caían, mientras que su hermana mayor estaba ocupada con la vaca y un corderito ciego. Al llegar, mientras la hermana mayor se sentaba en el pasto, cantando canciones religiosas en quechua, Felipe jugaba con los niños menores: trepaban a un árbol y jugaban a esconder el corderito bajo los arbustos, tiraban cosas y corrían a recogerlas. En todas estas actividades, Felipe usualmente tomaba la iniciativa y era rápidamente seguido por los más pequeños. De regreso, nuevamente estuvo a cargo de los más pequeños mientras su hermana mayor cuidaba a la vaca y la oveja. Felipe estaba asumiendo más tareas en las actividades domésticas y productivas de su hogar de un año a otro. Sin embargo, si el juego de Felipe lo distraía de la realización de sus tareas, su madre lo disciplinaba:

Mamá de Felipe: El otro año se dedicaba más a jugar señora, ahora ya hace algunas cositas... cargando leñitas, pela papa, habas señorita, así.

Entrevistadora: ¿Quién le ha enseñado?

Mamá de Felipe: Yo pues señora, «recoge apura», diciendo.

Entrevistadora: ¿Cualquier cosa que le digas hace? ¿O no hace?

Mamá de Felipe: Cuando le sobo con látigo sí hace señorita.

Entrevistadora: ¿Cuándo tú le pegas no más hace?

Mamá de Felipe: Ajá, si no hago, así a jugar se va.

La madre de Felipe explicó lo importante para su hijo de aprender habilidades domésticas y agrícolas:

Quizás cuando le hago estudiar no va a terminar, entonces así como nosotros no más va a vivir pues.

La participación progresiva de los niños en las actividades adultas, entonces, les permite adquirir las habilidades y desarrollar las competencias necesarias para vivir de la tierra, si la escolaridad no desemboca en un empleo en la ciudad.

Los casos de Ana y Felipe muestran que el género importa y que las niñas se involucran más temprano que los niños en las actividades del hogar. Los otros estudios de caso indicaron patrones similares a aquellos observados en los dos seleccionados como ejemplos. Como Felipe, Fabricio comenzó a ir a los campos con su padre para cosechar papas en el año que separó las dos visitas, y asumió más responsabilidad en el cuidado de las vacas. En el caso de Héctor, desde que 
su padre está ausente, acompaña a su hermano mayor a pastorear el rebaño y disfruta aprendiendo cosas de él. Mientras que las niñas, Flavia y Rosa, así como Ana, ayudan a sus madres con la cocina, la chacra y el pastoreo del rebaño y aprenden de ellas observando. Rosa era la hermana más joven de la familia pero Flavia tenía una hermana menor y cuidaba de ella como Ana hacía con la suya.

\section{3. Autonomía y participación en el mundo social: los niños mayores}

Los padres y madres de todas las comunidades enmarcaron la participación de los niños en las actividades domésticas y productivas como una práctica necesaria para su futuro, reconociendo no solo la importancia económica sino también la dimensión formativa de esta labor. Este proceso de involucramiento se inicia y consolida temprano en las vidas de los niños si lo comparamos con estándares urbanos. Hacia los 8 o 9 años ya abarcan un conjunto de conocimientos y destrezas y hacia los 12 o 13 años los niños y niñas ya tienen la mayor parte de las habilidades para participar como miembros plenos de su hogar.

Mamá de Felipe: Trabajan desde los nueve, doce años. (...) Es importante pues señora, con alguna cosita [dinero] te ayuda.

Entrevistadora: ¿Cómo tú te das cuenta que tu hijo ya está listo para trabajar? Mamá de Felipe: Él mismo pues me va a decir señora, según va creciendo, «ya voy a ir a trabajar» dice señorita.

Esta cita muestra el reconocimiento de las habilidades de los niños para contribuir económicamente al hogar desde los nueve o diez años, así como la autonomía que adquieren en la decisión de involucrarse en una actividad de esta índole. El trabajo entre los niños alcanza pues cierto nivel de competencia y se consolida como una fuente de provisión para las necesidades de la familia y de los propios niños, como lo recordó Sandro, de 12 años, en Andahuaylas:

Sandro: Está bien que los niños trabajen [en otras chacras]. Porque es fácil para pagar.

Entrevistadora: ¿Qué necesitan pagar? (...) ¿Necesitan pagar cosas?

Sandro: Sí. (...) Para examen (...) las fotocopias.

Las necesidades en estas comunidades involucran gastos en la escuela, como uniformes, materiales escolares, e incluso hojas de papel para imprimir los exámenes. Para permitir una continuidad de la escolaridad, los niños y sus familias tienen que asegurar la disponibilidad de este dinero. Como Sandro lo menciona, se trata de una tarea no solo de los padres sino también de sus hijos en edad escolar. De manera similar, Eva, una de las niñas mayores (13 años), señaló que el trabajo infantil ayuda a brindar recursos para las necesidades de los niños en tiempos de crisis del hogar: 
[cuando mi papá se enfermó] no había plata para nuestro pasaje así... nosotros nos íbamos a trabajar los sábados, o sea yo y mi hermano nada más.

Eva y su hermano estudiaban en un pueblo cercano y por ello necesitaban tomar el bus a diario. La enfermedad de su padre implicaba que no había dinero para el transporte, y por ello podrían dejar de asistir a la escuela. Pero en este caso los niños eran considerados suficientemente competentes para ir a trabajar en chacras ajenas por un pago y de esta manera proveer sus propias necesidades.

Sin embargo, el trabajo de los niños no remite solo a una estrategia de supervivencia sino también a un espacio para aprender y desarrollar competencias. En las comunidades se aprecia que el trabajo infantil se relacione con forjar relaciones afectivas e identidades de género. En discusiones grupales, los niños de 12 y 13 años declararon otorgarle un valor afectivo al trabajo en los campos como una manera de ser apreciados por el padre. Mientras tanto, las niñas de la misma edad enfatizaron su ayuda a las madres en el hogar y la madre de Ana también señaló cómo sus hijas aprenden observando mientras cocina o teje. En general, para todas las actividades domésticas y el trabajo en la chacra familiar, los niños en Andahuaylas usaban la frase «ayudar en la casa» y la diferenciaban del «trabajo» pagado en chacras ajenas, en el cual también participaban desde los 11 o 12 años (Alarcón [2011] encuentra una distinción similar en su trabajo en comunidades de Huancavelica). Esta actividad era considerada de manera positiva, como una forma de conseguir dinero en efectivo y pagar los útiles escolares, pero también podía ser vista como una fuente de preocupación en la medida en que se volviera muy pesada e implicara faltar a clases en la escuela.

Otro dato importante que proporcionaron los niños varones de 12 y 13 años fue su participación en eventos de carácter político-gremial. Poco antes de la primera visita había tenido lugar un paro agrario en su provincia y se había producido una concentración de campesinos en su comunidad. Los niños varones participaron junto a sus padres, y fueron testigos del enfrentamiento entre los manifestantes y la policía, que tuvo como resultado la muerte de un dirigente de una comunidad vecina. Los niños eran plenamente conscientes de los hechos ocurridos, incluso uno de ellos se había lastimado la pierna con un fragmento de los dispositivos lacrimógenos para dispersar a los manifestantes. Los niños vivían estos hechos como parte de su experiencia de acompañar a sus padres en los asuntos de la vida cotidiana que los atañen, de la misma forma en que los acompañaban a las chacras a trabajar. Puede vislumbrarse que no se trata pues solo de un tema de supervivencia, sino también de construir identidad y pertenencia a su grupo social.

\section{DISCUSIÓN: FORJANDO AUTONOMÍA, FORTALECIENDO RELACIONES}

En las secciones anteriores, hemos analizado los procesos a la vez de integración de los niños y niñas andinos a su cultura, y de interiorización de saberes y destrezas que los convierten en miembros plenos de la misma. Así, hemos evidenciado la 
forma como los niños quechuas rurales en Perú experimentan diversos cambios en sus primeros años de vida: su llegada a la familia (recibir un nombre), dejar de ser un bebé (a través del primer corte de pelo), y asumir mayores responsabilidades en el hogar (al iniciar la niñez intermedia). Mientras que los dos primeros están usualmente marcados por una ceremonia, el tercero implica un proceso menos visible, más progresivo y claramente identificado por los adultos. El aprendizaje y la interacción social son claves en estos procesos de cambio, pues están asociados no necesariamente con la edad cronológica o la apariencia física, sino con el tipo de habilidades y capacidades que los niños pueden manifestar en diferentes momentos de sus vidas y con las cambiantes expectativas de los adultos y de otros niños a su alrededor.

En todas las comunidades estudiadas, los niños y niñas comparten el tipo de experiencias que hemos ilustrado a partir de casos específicos. Demuestran altos niveles de autonomía y al mismo tiempo de colaboración con la vida familiar, lo que se incrementó notablemente de un año al siguiente en el caso de Andahuaylas, donde pudimos observar el tránsito hacia la niñez intermedia. Este es reconocido por los niños y sus padres, y corresponde con las expectativas de los adultos en cada comunidad en términos de responsabilidades domésticas como el cuidado de los hermanos menores y la ayuda con la agricultura o la crianza del ganado. En todas las comunidades, los niños acompañan a sus padres al trabajar sus chacras desde la primera infancia. Cerca de los cinco años ya desempeñaban algunas actividades agrícolas con autonomía, mientras que aprendían otras habilidades observando a sus hermanos mayores o a los adultos.

Esta manera de aprender ha sido caracterizada como aprendizaje por «observación y ayuda» pero, en contraste con las interpretaciones usuales que consideran la observación como una conducta pasiva, Paradise \& Rogoff han enfatizado que «la observación es un proceso activo, que acompaña o se anticipa a contribuir con eventos de importancia en la familia y la comunidad» (2009: 104). Este tipo de aprendizaje es facilitado en sociedades donde los niños se socializan con el mundo de los adultos (en vez de estar separados de él), y donde los adultos participan en el trabajo organizado dentro del hogar y en la comunidad inmediata, pues es más probable que los niños estén a su alrededor (Gaskins \& Paradise, 2010).

Dado que los niños y niñas participan desde una edad temprana en las tareas familiares y comunitarias, forman parte de una realidad económica y social compartida con los adultos (Paradise \& Rogoff, 2009). Como parte de este proceso, las diferencias de género van apareciendo a lo largo de la infancia en las comunidades estudiadas, lo que se evidencia en su vestimenta, las herramientas que usan y las actividades en las que participan en mayor medida, que van asemejándose a las del padre o la madre según se trate de un niño o niña respectivamente.

En todas las comunidades, los adultos y niños valoran la participación infantil en las actividades familiares y su creciente responsabilidad. Durante las entrevistas, los niños pequeños reportaban su gusto por las tareas aprendidas. Al observarlos en el hogar, era evidente que se enorgullecían de las cosas que podían hacer, y en 
sesiones grupales relataron y dibujaron con entusiasmo todas las cosas que eran capaces de hacer para ayudar. Ello indica la representación de los niños acerca de sus actividades como forma de participación en los deberes a desempeñar para el bienestar de su familia. Otros autores han señalado que de modo general los niños adquieren autoestima cuando se sienten competentes, respetados y valorados por sus contribuciones al hogar, y su sentimiento de valoración de sí mismos mejora (Woodhead, 2007). Los niños mayores solían elaborar un punto de vista sobre sus actividades, expresando opiniones positivas sobre el trabajo como factor de aprendizaje, autonomía, responsabilidad y adquisición de habilidades prácticas, así como fuente de ingresos. En ese sentido, la participación económica de los niños en estas comunidades puede ser caracterizada como una forma tanto de supervivencia como de socialización (Woodhead, 2007). Esto significa que los niños no solo reconocen la necesidad de su trabajo a largo plazo para el bienestar de la familia, sino que también lo ven como una oportunidad para adquirir habilidades adultas valoradas por ellos mismos y por su grupo social.

Tanto los padres como los hijos consideran la participación de los niños en las actividades productivas como maneras de obtener el conocimiento, las destrezas y las habilidades que los ayudarían a ganarse la vida en el futuro - tanto como complemento de sus carreras escolares o en vez de ellas si la escuela fallaba al brindarles acceso a empleos urbanos- Sin embargo, los aspectos prácticos de la participación de los niños en las tareas familiares son solo una parte del proceso: la preocupación gira también alrededor del desarrollo de la responsabilidad según los principios de obligaciones mutuas entre miembros de la misma familia y más allá del círculo doméstico. La importancia de estas relaciones se evidencia en las celebraciones culturales como la asignación del nombre, el primer corte de pelo y el bautismo católico. Los niños mayores también son conscientes de esta dimensión «moral» de sus actividades al afirmar que el trabajo ayuda a los niños a evitar volverse «flojos» o al valorar y asociar la obediencia con el cumplimiento de las tareas domésticas. En este punto, la adquisición de conocimientos social y económicamente relevantes hace que los niños aprendan al mismo tiempo cómo participar recíprocamente como miembros responsables de un grupo social, ganando no solo habilidades prácticas sino también conocimiento sociocultural (Paradise \& de Haan, 2009: 197). De manera similar, Ochs \& Izquierdo (2009), enfocadas en el desarrollo moral, han propuesto que la participación de los niños en actividades domésticas no solo ofrezca competencias prácticas, sino que también promueva responsabilidad moral, pues crea consciencia social, capacidad de respuesta a las necesidades ajenas y autosuficiencia. Por ello, se podría sugerir que, además de las habilidades, en estas comunidades se adquiere un sentido de responsabilidad moral de manera temprana, a través de los procesos descritos y el aprendizaje que la acompaña. Asimismo, notamos en los niños y niñas no solo el desarrollo de habilidades domésticas y laborales, o de responsabilidad al llevarlas a cabo, sino también un sentimiento de pertenencia al grupo en el cual los niños participan, lo cual a su vez se expresa en una identidad compartida. Así, la participación en este tipo de actividades permite el fortalecimiento de una relación de identidad con la familia y la comunidad (Paradise \& De Haan, 
2009). La participación infantil en estas actividades se relaciona entonces con su bienestar general y con un sentido de identidad dentro de su grupo social, lo que ayuda a entender las opiniones positivas sobre el trabajo de los niños referidas previamente.

\section{CONCLUSIÓN}

La incorporación de los niños andinos a su cultura se inicia desde su nacimiento y a lo largo de sus primeros años de vida, a través de su participación gradual en diversas actividades cotidianas de sus ámbitos sociales. Asimismo, los niños y niñas asumen un conjunto de tareas que van desempeñando con creciente autonomía y destreza. En ese proceso adquieren un sentido de responsabilidad moral, que les conecta con las necesidades de otros miembros de la familia y les permite responder en consecuencia. Paralelamente, desarrollan un conjunto de relaciones sociales que les recuerda constantemente que su mundo social está tejido por los intercambios producidos en ellas. Esta socialización y las responsabilidades que asumen les permiten interiorizar los conocimientos y habilidades necesarias para la vida cotidiana. En esta dinámica se juega además la posibilidad de construir una identidad compartida, en la cual se reconocen como miembros de un colectivo específico. Afirmamos que este proceso es «temprano» en la medida en que sucede entre los 5 y 8 años de edad. Los niños mayores de 12 y 13 años ya son participantes plenos de la mayor parte de las actividades domésticas, económicas e incluso políticas de su comunidad. En los años siguientes algunos migrarían por trabajo, otros continuarían estudiando, unas más serían madres. El final de la infancia parecía más cerca para muchos de ellos, obligándonos a repensar las definiciones comúnmente aceptadas de infancia y los umbrales etarios en los que se transita a la adultez, aunque los proyectos de escolarización de sus familias podrían contribuir a extender estos tiempos. La riqueza de los lazos y compromisos sociales que se van tejiendo en la vida de los niños andinos nos reta a verlos no como individuos aislados (como muchas veces representamos al «niño») sino como participantes plenos de un mundo de relaciones y responsabilidades.

\section{Referencias citadas}

ALARCÓN, W., 2011 - Trabajo infantil en los Andes, 136 pp.; Lima: IEP, Fundación Proyecto Solidario por la Infancia, Telefónica.

AMES, P., 1999 - El poder en el aula: un estudio en escuelas rurales andinas. In: El poder visto desde abajo. Democracia, educación y ciudadanía en espacios locales (M. Tanaka, ed.): 267-334; Lima: Instituto de Estudios Peruanos.

AMES, P., 2001 - ¿Libros para todos? Maestros y textos escolares en el Perú rural, 85 pp.; Lima: Consorcio de Investigaciones Económicas y Sociales, Instituto de Estudios Peruanos. 
AMES, P., 2002 - Para ser iguales, para ser distintos. Educación escritura y poder en el Perú, 111 pp.; Lima: Instituto de Estudios Peruanos.

AMES, P., 2013 - Learning to be responsible: Young children transitions outside school. Learning, Culture and Social Interaction, 2 (3): 143-154.

BANCO MUNDIAL, 2013 - http://datos.bancomundial.org/pais/peru

BOLIN, I., 2006 - Growing up in a culture of respect. Child rearing in the highlands of Peru. 232 pp.; Austin, TX: University of Texas Press.

BOURDILLON, M., 2006 - Children and work: A review of current literature and debates. Development and Change, 37: 1201-1226.

BOYDEN, J., MYERS, W. \& LING, B., 1998 - What Works for Working Children?, 364 pp.; Stockholm: Rädda Barnen.

CAVAGNOUD, R., 2011 - Entre la escuela y la supervivencia. Trabajo adolescente y dinámicas familiares en Lima, 438 pp.; Lima: IFEA, IEP, FT.

DEL PINO, P., MENA, M., TORREJON, S., DEL PINO, E., ARONES, M. \& PORTUGAL, T., 2012 - Repensar la desnutrición: infancia y cultura en Ayacucho, Perú, 250 pp.; Lima: Instituto de Estudios Peruanos.

GAITÁN MUÑOZ, L., 2006 - La nueva sociología de la infancia. Aportaciones de una mirada distinta. Política y Sociedad, 43 (1): 9-26.

GARCÍA, F., 2005 - Yachay. Concepciones sobre la enseñanza y aprendizaje en una comunidad quechua, 135 pp.; La Paz: PINSEIB, PROEIB Andes, Plural Editores.

GASKINS, S. \& PARADISE, R., 2010 - Learning through observation in daily life. In: The anthropology of learning in childhood (D. F. Lancy, S. Gaskins, S. \& J. Bock, eds.): 85-117; Lanham: Alta-Mira Press.

INSTITUTO NACIONAL DE ESTADÍSTICA E INFORMÁTICA-INEI, 2008 - Censos nacionales 2007: XI de población y VI de vivienda. Lima: INEI.

INSTITUTO NACIONAL DE ESTADÍSTICA E INFORMÁTICA-INEI, 2010a - Encuesta Nacional de Hogares 2009. Principales resultados. Lima: INEI.

INEI, 2010b - Informe técnico: Evolución de la pobreza 2009.

http://censos.inei.gob.pe/DocumentosPublicos/Pobreza/2009/Infome_Pobreza.pdf (último acceso 2/11/2011).

ISBELL, B. J., 1997 - De inmaduro a duro: lo simbólico femenino y los esquemas andinos de género. In: Parentesco y género en los Andes, Tomo I: Más allá del silencio: las fronteras de género en los Andes (D. Arnold, ed.): 253-301; La Paz: CIASE, ILCA.

JAMES, A. \& PROUT, A. (eds), 1997 - Constructing and reconstructing childhood, 206 pp.; Londres: Routledge Falmer.

JOHNSON-HANKS, J., 2002 - On the limits of life stages in ethnography: Toward a theory of vital conjunctures. American Anthropologist, 104: 865-880.

LANCY, D., 2010 - Learning "from nobody": The limited role of teaching in folk models of children's development. Childhood in the Past, 3: 79-106.

LANCY, D. \& GROVE, A., 2011 - Middle childhood in cross-cultural perspective. Human Nature, 22: 281-302.

LAVE, J. \& WEGNER, E., 1991 - Situated learning: Legitimate peripheral participation, 132 pp.; Cambridge: Cambridge University Press.

LESTAGE, F., 1999 - Naissance et petite enfance dans les Andes péruviennes : pratiques, rites, représentations, 300 pp.; París-Montreal: Éditions L'Harmattan.

OCHS, E. \& IZQUIERDO, C., 2009 - Responsibility in Childhood: Three Developmental Trajectories. Ethos, 37 (4): 391-413. 
ORTIZ, A., 2001 - La pareja y el mito, 420 pp.; Lima: PUCP.

PARADISE, R., 2011 - ¿Cómo educan los indígenas a sus hijos? El cómo y el por qué del aprendizaje en la familia y en la comunidad. In: Aprendizaje, cultura y desarrollo: Una aproximación interdisciplinaria (S. Frisancho, M. T. Moreno, P. Ruiz Bravo \& V. Zavala, eds.): 41-60; Lima: PUCP.

PARADISE, R. \& DE HAAN, M., 2009 - Responsibility and reciprocity: Social organization of Mazahua learning practices. Anthropology and Education Quarterly, 40: 187204.

PARADISE, R. \& ROGOFF, B., 2009 - Side by side: Learning by observing and pitching in. Ethos, 37: 102-138.

PLATT, T., 2001 - El feto agresivo. Parto, formación de la persona y mito-historia en los Andes. Anuario de Estudios Americanos, 58, 2: 633-678.

ROGOFF, B., 1990 - Apprenticeship in thinking: Cognitive development in social context, 242 рр.; New York: Oxford University Press.

ROGOFF, B., 1996 - Developmental Transitions in Children's Participation in Sociocultural Activities. In: The five to seven year shift: The age of reason and responsibility (A. Sameroff \& M. Haith, eds.): 273-294; Chicago: University of Chicago Press.

ROMÁN DE SILGADO, M., 1990 - Ciclo vital en las comunidades andinas. In: La escuela rural: Variaciones sobre un tema (C. Montero, ed.): 275-278; Lima: Ministerio de Agricultura, Ministerio de Educación, FAO, Cotesu.

SPINDLER, G., 1993 - La transmisión de la cultura. In: Lecturas de antropología para educadores (Velasco Maillo et al., eds.): 205-241; Madrid: Trota.

SUREMAIN, C.-E. 2010 - Quand « le cheveu fait l'homme ». La cérémonie de la première coupe de cheveux de l'enfant en Bolivie. Autrepart, 55: 125-140.

TRONICK, E. Z., THOMAS, R. B. \& DALTABUIT, M., 1994 - The Quechua manta pouch: A caretaking practice for buffering the Peruvian infant against the multiple stressors of high altitude. Child Development, 65: 1005-1013.

UNICEF, 2010 - Estado de la niñez indígena en el Perú, 152 pp.; Lima: UNICEF, INEI.

VAN GENNEP, A., 1960 - The rites of passage, 198 pp.; Chicago, IL: University of Chicago Press.

VOGLER, P., CRIVELLO, G. \& WOODHEAD, M., 2008 - Early childhood transitions research: A review of concepts, theory, and practice, Working Paper No. 48, The Hague: Bernard van Leer Foundation.

WOODHEAD, M., 2007 - Harmed by work or developing through work? Issues in the study of phycosocial impacts. In: Working to be someone: Child focused research and practice with working children (B. Hungerland, M. Liebel, B. Milne, \& A. Wihstutz, eds.): 31-42; London: Jessica Kingsley. 